\title{
Large Concha Bullosa Is a Risk Factor for Chronic Sinusitis: A Case Control Study
}

\author{
Alexis Do Santos Zounon ${ }^{1,2, *}$, Ulrich Bidossessi Vodouhe ${ }^{1}$, Jean-Baptiste Agai ${ }^{3}$, Djibril Balde ${ }^{4}$, \\ Sonia Adjanohoun ${ }^{1}$, Wassi Adjibabi ${ }^{1}$, Bernadette Vignikin-Yehouessi ${ }^{1}$ \\ ${ }^{1}$ Department of Health Sciences, University of Abomey-Calavi, Cotonou, Benin \\ ${ }^{2}$ ENT and Cervico Facial Surgery Department, Military University Hospital, Cotonou, Benin \\ ${ }^{3}$ Department of Radiology, Military University Hospital, Cotonou, Benin \\ ${ }^{4}$ ENT and Cervico Facial Surgery Department, Regional Hospital Heinrich Lubke, Diourbel, Senegal
}

Email address:

azdosantos@yahoo.fr (A. Do S. Zounon)

${ }^{*}$ Corresponding author

\section{To cite this article:}

Alexis Do Santos Zounon, Ulrich Bidossessi Vodouhe, Jean-Baptiste Agai, Djibril Balde, Sonia Adjanohoun, Wassi Adjibabi, Bernadette Vignikin-Yehouessi. Large Concha Bullosa Is a Risk Factor for Chronic Sinusitis: A Case Control Study. International Journal of Otorhinolaryngology. Vol. 5, No. 2, 2019, pp. 35-38. doi: 10.11648/j.ijo.20190502.11

Received: June 26, 2019; Accepted: July 17, 2019; Published: July 31, 2019

\begin{abstract}
The study analyzed the correlation between the presence of concha bullosa with the presence of radiological sinus opacity and sinonasal functional symptoms. All patients whose computed tomography (CT) findings were positive for concha bullosa were included in the study. The CT parameters taken in consideration were the presence and volume of concha bullosa, the aspect of the ipsilateral maxillary sinus (normal transparency, presence of sinus opacity). Clinical parameters were sinonasal functional signs. Statistical comparisons were made using the Pearson Chi square test. Over a four years period, 2436 CTs were interpreted, 276 of which presenting a concha bullosa (prevalence of $11.33 \%$ ). The average age was 33 (19 to 63). Females (204 either 74\%) outnumbered men (72 either 26\%). An opacification of the ipsilateral maxillary sinus seen upon CT was statistically related to the presence of a large concha bullosa $(p=0.02)$. On the other hand, the presence of a clinical symptomatology of maxillary sinusitis crossed with the presence of a large concha bullosa was not significant ( $\mathrm{p}>0.50)$. This study has demonstrated a close correlation between the existence of a large concha-bullosa and an ipsilateral maxillary sinusitis seen on CT-Scan without necessarily having clinical manifestation of sinusitis. The diagnosis of sinusitis must remain primarily clinical.
\end{abstract}

Keywords: Concha Bullosa, Sinusitis, Opacified Sinus

\section{Introduction}

Concha bullosa (CB) is a pneumatization of the middle nasal turbinate from anterior ethmoid cell [1]. It is present in almost half of the population [2]. According to P. Lehmann and al., it is the most common accessory sinonasal pneumatization and is present in more than $30 \%$ of the population [3]. It is described as an anamical variation that can induce a confinement of the ipsilateral maxillary sinus by narrowing of the ostiomeatal complex [4-6]. It is therefore a malformation, exposing the ipsilateral maxillary sinus to risks of infection [7-9]. This risk is still debated by several other authors $[10,11]$.
The aim of the present study is to analyze the correlation between the presence of a concha bullosa with both the existence of radiological sinus opacity and sinonasal functional signs.

\section{Material and Method}

This is a cross-sectional, descriptive and analytical study covering a four-year period from January 2nd, 2015 to December 31st, 2018, carried out at the military university hospital in Cotonou. Computed tomography (CT) examinations were performed at the Military University Hospital of Cotonou's Radiology Department. All CT scans 
performed during the study period and showing the sinonasal complex were examined. Patients whose CT images had objectified a concha bullosa were included. Patients whose sinonasal anatomic structure had been affected by tumor, trauma or other malformation were excluded from the study

CT and clinical data from each patient included in the study were reported on investigation forms. The clinical parameters were the presence or absence of sinonasal functional symptomatology. The CT parameters were the presence and the type of concha bullosa, the aspect of the ipsilateral maxillary sinus (normal transparency, presence of sinus opacity). Concha bullosa (CB) has been defined by pneumatization of the middle turbinate regardless of its extent. In case of bilateral $\mathrm{CB}$, the dominant concha was assessed for the study. It was defined as "small" when the vertical height was less than $50 \%$ of the total height of the middle turbinate, measured on the coronal sections. It was been described as "large" when its vertical height was greater than $50 \%$ with a subjective average increase in the overall volume of the middle turbinate. CT scans were performed with an 8 cameras GE Brightspeed 16 slices Service 192.9.101.1 GDAS 16.

Microsoft Excel software was used for data entry. Stata software version 11 was used for data analysis. Statistical comparisons of qualitative variables were made using the Pearson Chi square test at a significance level of $5 \%$.

\section{Result}

\subsection{Epidemiology}

Over four years 2436 CT have been interpreted. 276 files were included in the study presenting either a unilateral or bilateral concha bullosa. This represents a hospital prevalence of $11.33 \%$. All the patients were adults with an average age of 33 (19 to 63 years). Females (204 either 74\%) outnumbered men (72 either $26 \%$ ), a sex ratio of 0.35 .

\subsection{Characteristics of $C B$}

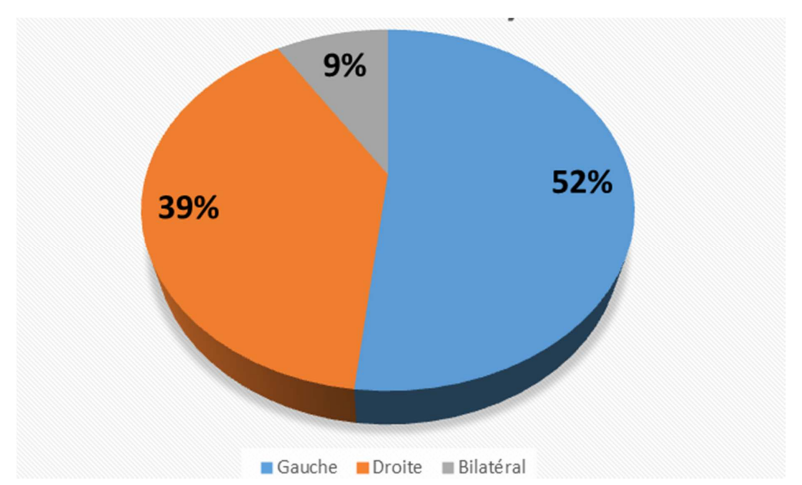

Figure 1. Distribution of concha bullosa cases based on their laterality.

Legend: blue for left sided CB (52\%); orange for right sided CB (39\%); grey for bilateral CB $(9 \%)$.

Among the 276 patients with $\mathrm{CB}$, left side $\mathrm{CB}$ were more frequent (174 either 63\%) than right side $\mathrm{CB}(132 ; 48 \%)$. Thirty (30 either $22 \%$ ) patients had bilateral $\mathrm{CB}$ and were classified in both left side and right side groups.

\subsection{Size}

Large CB $(222 ; 80 \%)$ were more frequently seen than small ones $(54 ; 20 \%)$

\subsection{Transparence of Sinuses}

All types of ipsilateral maxillary sinus opacification were mentioned. Sinus wall thickening were most frequent (120; $43.5 \%)$.

Table 1. Distribution of ipsilateral maxillary sinus CT findings based on Concha Bullosa size.

\begin{tabular}{llll}
\hline & Opacified sinus & Normal transparence & Total \\
\hline Large CB & $162(58,70 \%)$ & $60(21,74 \%)$ & $222(80,4 \%)$ \\
Small CB & $18(6,52 \%)$ & $36(13,04 \%)$ & $54(19,6 \%)$ \\
Total & $180(65,2 \%)$ & $96(34,8 \%)$ & $276(100 \%)$ \\
\hline
\end{tabular}

The relationship between ipsilateral maxillary sinus opacity and the existence of a Concha Bullosa was statistically significant $(\mathrm{p}=0,02)$.

\subsection{Clinical Symptomatology}

Symptomatic ipsilateral maxillary sinusitis was crossed with the size of concha bullosa. Clinical sinusitis was not statistically linked with the existence of a large concha bullosa $(\mathrm{p}>0,50)$.

Table 2. Distribution of clinical expression of maxillary sinusitis based on ipsilateral concha bullosa size.

\begin{tabular}{llll}
\hline & Clinical sinusitis & No clinical sinusitis & Total \\
\hline Large CB & 42 & 180 & $222(80,4 \%)$ \\
Small CB & 6 & 48 & $54(19,6 \%)$ \\
Total & $48(17,4 \%)$ & $228(82,6 \%)$ & $276(100 \%)$ \\
\hline
\end{tabular}

\section{Discussion}

The prevalence of concha bullosa is highly variable in the population, estimated at more than 50\% [12]. The present study found a low prevalence $(11.33 \%)$ which is similar to severahgxwl other publications [13]. The predominance of concha bullosa in young adult females is described in the litterature [2] and our results correlate this fact.

The study did not focus on patients with a sinonasal complaint, but embraced all patients who had CT scans showing the sinonasal complex, including orbital, cerebral, facial, and pharyngeal CT scans. This prevented the search for concha bullosa in patients with rhinological symptoms alone as this could be a recruitment bias. The results could therefore be more easily applied onto the general population. In the current series, a predominance on the left has been noted. Only 30 patients, or $22 \%$ of the series had bilateral involvement. These results are similar to those obtained by Saarthak W et al [14] who also described a predominance of left side Concha Bullosa and a low rate of bilaterality. Jamie S. et al [2] studies have highlighted the rarity of bilateral lesions. There is no argument explaining this. However, 
Saarthak W [14] suggested that this could vary from one ethnic group to another and be dependent on pneumatization parameters. This argument adds to the importance of the present study carried out on an exclusively black African study population.

$65.2 \%$ of the patients presented an opacified ipsilateral maxillary sinus, among them $43.1 \%$ presented a thickening of the wall frame and $22.1 \%$ a diffuse filling. Broad concha bullosa was more frequent, accounting for $80 \%$ of patients. This result is similar to that of Jamie $\mathrm{S}$ et al [2] who found an estimated distribution of $58 \%$ of large concha bullosa. The presence of ipsilateral sinus opacity was statistically significant in patients with large concha bullosa $(\mathrm{p}=0.02)$, suggesting a link between the presence of a large concha bullosa and the occurrence of a radiologically visible sinusitis. These results could be explained by the fact that the concha bullosa of significant size induces a confinement of the ipsilateral maxillary sinus by narrowing of the ostiomeatal complex [4-6] thus decreasing aeration of the sinus and consequently its inflammation. Shin HS [7] and Calhoun $\mathrm{KH}$ et al [8] have stated in their studies that there is a relationship between concha bullosa and sinusitis. Jamie $\mathrm{S}$ [2] found no significant correlation. Duran Karatas' team demonstrated a positive correlation between the volumes of concha bullosa and paranasal sinus [15]. Anatomic variants such as concha bullosa may therefore have a proven influence on patients with chronic rhino sinusitis [16].

Clinically, only 48 patients $(17.4 \%)$ had clinical signs of sinusitis. We found no correlation between the presence of concha bullosa, even large ones, with the presence of clinical signs of sinusitis $(p>0.50)$. This is a reminder of the importance of clinical manifestations rather than radiological signs when taking a therapeutic decision. Diagnosing sinusitis can be challenging in some cases; and the combination of clinical manifestations and endoscopic results may not be able to predict CT findings [17]. Considering the high cost of paraclinical exams in our context, CT scanning is done only in case of atypical findings, for further assessment, or in case of unfavorable evolution under treatment.

\section{Conclusion}

Middle turbinate pneumatization is a common anatomical variation typically discovered incidentally when a patient undergoes CT Scan of the sinuses. This study showed the presence of a close correlation between the existence of a large concha bullosa and an ipsilateral maxillary sinusitis on imaging. This should not require treatment since there are no clinical symptomatology. Indeed the study shows that there is no statistical relationship between a large concha bullosa and the sinus disease. The diagnosis of sinusitis must still primarily be based on clinical findings.

\section{Conflict of Interest}

All the authors do not have any possible conflicts of interest.

\section{References}

[1] Marsot-dupuch. K, Genty E Les variantes Anatomiques des Sinus de la Face. J RADIOL 2003; 84: 357-367.

[2] Jamie S. Stallman, Joao N. Lobo, and Peter M. Som. The Incidence of Concha Bullosa and Its relationship to Nasal Septal Deviation and Paranasal Sinus Disease. AJNR Am J Neuroradiol. 2004; 25: 1613-1618.

[3] P Lehmann, R Bouaziz, C Page, M Warin, G Saliou, B Deschepper, V Strunski, H Deramond. Cavités sinusiennes de la face: aspect scanographique des variantes anatomiques et leur risque chirurgical. J Radiol. 2009; 90: 21-30.

[4] Livre «Imagerie en ORL» Frédérique Dubrulle ElsevierMasson, 2010 (ISBNh 978-2-294-70498-7).

[5] Mcdonnell D, Esposito M, Todd ME (1992) A teaching model to illustrate the variation in size and shape of the maxillary sinus. J Anat 181: 377-380.

[6] Riellol APL and Boasquevisque EM (2008) Anatomical variants of the osteomeatal complex: tomographic findings in 200 patients. Radiol Bras 41 (3): 149-154.

[7] Shin HS. Clinical significance of unilateral sinusitis. J Korean Med. Sci 1986; 1: 69-74.

[8] Calhoun KH, Waggenspack GA, Simpson CB, et al. CT evaluation of the paranasal sinuses in symptomatic and asymptomatic populations. Otolaryngol Head Neck Surg 1991; 104: 480-483.

[9] Anukaran Mahajan, Anupama Mahajan, Karunesh Gupta, Pankaj Verma. Anatomical Variations of Osteomatal Complex: An Endoscopic Study. Anatomy Physiol Biochem Int J: 2018; 5 (2): 555659. DOI: 10.19080/APBIJ.2018.05.555660.

[10] Lloyd G, Lund V, Scadding G. CT of the paranasal sinuses and functional endoscopic surgery: a critical analysis of 100 symptomatic patients. J Laryngol Otol 1991; 105: 181-185.

[11] Lloyd GA. CT of the paranasal sinuses: study of a control series in relation to endoscopic sinus surgery. J Laryngol Otol 1990; 104: 477-81.

[12] Fadda GL, Rosso S, Aversa S, Petrelli A, Ondolo C, Succo G. Multiparametric statistical correlations between paranasal sinus anatomic variations and chronic rhinosinusitis. Acta Otorhinolaryngol Ital 2012; 32: 244-51.

[13] Soo Kweon Koo, Jong Deok Kim, Ji Seung Moon, Sung Hoon Jung, Sang Hoon Lee. The incidence of concha bullosa, unusual anatomic variation and its relationship to nasal septal deviation: A retrospective radiologic study. Auris Nasus Larynx (2017), http://dx.doi.org/10.1016/j.anl.2017.01.003.

[14] Saarthak W, Naveen S, Uma G, Prishni D. Concha bullosa: types and relationship with chronic sinusitis. International Journal of Otorhinolaryngology and Head and Neck Surgery. $2017 \mathrm{Jul} ; 3$ (3): 482-485.

[15] Duran Karatas; Fatih Yüksel; Ali Koç. Volumetric correlation between concha bullosa and paranasal sinuses. Journal of the Anatomical Society of India 2017; 66 (2): 131-134. https://doi.org/10.1016/j.jasi.2017.05.011. 
[16] Johnny Wu; Ravi Jain; Richard Douglas. Effect of paranasal anatomical variants on outcomes in patients with limited and diffuse chronic rhinosinusitis. Auris Nasus Larynx 2017; 44 (4): 417-421. https://doi.org/10.1016/j.anl.2016.08.009.
[17] Paige Moore, Brian Blakley, Eric Meen. Clinical predictors of chronic rhinosinusitis: do the Canadian clinical practice guidelines for acute and chronic rhinosinusitis predict CTconfirmation of disease? Journal of Otolaryngology - Head and Neck Surgery 2017; 46: 65. 